\title{
Correction to: Assessment of knee pain from MR imaging using a convolutional Siamese network
}

\author{
Gary H. Chang ${ }^{1}$ - David T. Felson ${ }^{2,3}$ - Shangran Qiu ${ }^{1}$ - Ali Guermazi ${ }^{4}$. Terence D. Capellini ${ }^{5,6}$. \\ Vijaya B. Kolachalama ${ }^{1,7,8,9}$
}

Published online: 22 July 2020

(C) European Society of Radiology 2020

\section{Correction to: European Radiology (2020) 30:3538-3548 https://doi.org/10.1007/s00330-020-06658-3}

The original version of this article, published on 13 February 2020, unfortunately contained a mistake. The Electronic Supplementary Material was missing in the online version. The ESM is available in the online version of this correction article.

Publisher's note Springer Nature remains neutral with regard to jurisdictional claims in published maps and institutional affiliations.

The online version of the original article can be found at https://oi.org/ $10.1007 / \mathrm{s} 00330-020-06658-3$

Electronic supplementary material The online version of this article (https://doi.org/10.1007/s00330-020-07073-4) contains supplementary material, which is available to authorized users.

Vijaya B. Kolachalama

vkola@bu.edu

1 Section of Computational Biomedicine, Department of Medicine, Boston University School of Medicine, 72 E. Concord Street, Evans 636, Boston, MA 02118, USA

2 Section of Rheumatology, Department of Medicine, Boston University School of Medicine, Boston, MA 02118, USA

3 Centre for Epidemiology, University of Manchester and the NIHR Manchester BRC, Manchester University, NHS Trust, Manchester, UK
4 Department of Radiology, Boston University School of Medicine, Boston, MA 02118, USA

5 Department of Human Evolutionary Biology, Harvard University, Cambridge, MA 02138, USA

6 Broad Institute of MIT and Harvard, Cambridge, MA 02142, USA

7 Whitaker Cardiovascular Institute, Boston University School of Medicine, Boston, MA 02118, USA

8 Hariri Institute for Computing and Computational Science and Engineering, Boston University, Boston, MA 02215, USA

9 Boston University Alzheimer's Disease Center, Boston, MA 02118, USA 